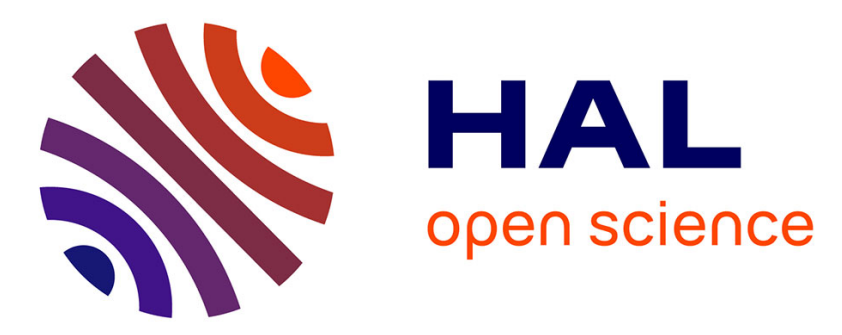

\title{
Discrete mode observability of switching linear systems with unknown inputs. a graph-theoretic approach
}

Taha Boukhobza, Frédéric Hamelin, Mohamed Ghassane Kabadi, Samir

Aberkane

\section{- To cite this version:}

Taha Boukhobza, Frédéric Hamelin, Mohamed Ghassane Kabadi, Samir Aberkane. Discrete mode observability of switching linear systems with unknown inputs. a graph-theoretic approach. 18th IFAC World Congress, IFAC WC'2011, Aug 2011, Milan, Italy. pp.6616-6621, 10.3182/20110828-6IT-1002.01005 . hal-00650662

\section{HAL Id: hal-00650662 https://hal.science/hal-00650662}

Submitted on 12 Dec 2011

HAL is a multi-disciplinary open access archive for the deposit and dissemination of scientific research documents, whether they are published or not. The documents may come from teaching and research institutions in France or abroad, or from public or private research centers.
L'archive ouverte pluridisciplinaire HAL, est destinée au dépôt et à la diffusion de documents scientifiques de niveau recherche, publiés ou non, émanant des établissements d'enseignement et de recherche français ou étrangers, des laboratoires publics ou privés. 


\title{
Discrete mode Observability of switching linear systems with unknown inputs. A graph-theoretic approach
}

\author{
T. Boukhobza, F. Hamelin, G. Kabadi, and S. Aberkane \\ Centre de Recherche en Automatique de Nancy (CRAN), Nancy-University, CNRS UMR 7039 \\ email: taha.boukhobza@cran.uhp-nancy.fr
}

\begin{abstract}
This paper deals with the observability of the discrete mode of switching structured linear systems with unknown inputs. The proposed method, based on a graph-theoretic approach, assumes only the knowledge of the system's structure. We express, in graphical terms sufficient conditions for the generic observability of the discrete mode. If these conditions are not satisfied, we also propose a sensor placement procedure which allows to recover the mode observability. More precisely, we provide an exhaustive location set for additional sensors to recover the given sufficient mode observability condition. Our approach uses algorithms which have polynomial complexity orders and assumes only the knowledge of the system's structure. It can be implemented by classical graph-theory algorithms.
\end{abstract}

Keywords: Switching linear systems, mode observability, sensor location, graph theory.

\section{INTRODUCTION}

Hybrid systems, combining event-driven and time-driven dynamics, have received growing attention in the control community as they can be used to describe a wide range of physical and engineering systems $[11,1,16]$. For such systems, the general problem of estimating the mode, the internal state and the unknown input is of great interest in many control areas. In the context of switching continuous time linear systems, studies presented in $[3,7,8,20]$ are among the most significant works which deal with observability. They characterize completely the observability of the continuous internal state and/or the discrete mode variable, which is assumed to be unknown and arbitrary. Other definitions of observability for other kinds of hybrid systems are also provided in the literature. For instance, $[4,6,5]$ study the observability of hybrid linear systems, where the discrete mode depends on the state trajectory or is associated to discrete outputs. Deterministic discrete-time switching linear systems were considered in $[19,2,4]$, knowing that, contrary to classical linear systems, important differences exist between the discrete and continuous time cases for switching linear systems (SLS) [3]. Even if our study is only concerned by mode observability, From the point of view of the class of systems under study and observability definitions used here, our paper is close to $[3,7,20,8]$. But, our proposed method and the obtained results present two main originalities. On the one hand, few works deal with the observability of hybrid systems with unknown inputs, which model disturbances or faults for example. In fact, there exist some works ([13] and the references therein), which aim at designing unknown input observers for SLS, but they do not study state and input observability. [21] addresses the problem of recovering the discrete mode variable and the input given an output and an initial state but not simultaneous state and input observability. On the other hand, in most cases, observability analysis for SLS deals with algebraic and geometric tools and so requires the exact knowledge of the state space matrices characterizing the systems' model. In many modeling problems or during conception stage, these matrices have a number of fixed zero entries determined by the physical laws while the remaining entries are not precisely known. In these cases, to study the structural properties, like observability, the idea is that we only keep the zero/non-zero entries in the state space matrices. Thus, we consider models where the fixed zeros are conserved while the non-zero entries are replaced by free parameters. Many interesting works on these models, called structured models, are related to the graph-theoretic approach and aim to analyse properties such as controllability, observability ... [9]. It results from these works that the graphtheoretic approach provides quite simple and elegant analysis tool.

In this context, the first part of the paper is proposed a graphbased approach to provide sufficient conditions which ensure the discrete mode observability for switching structured linear systems (SSLS). In a second part, and using a graph-theoretic approach, we address the problem of sensor placement in order to recover discrete mode observability property. More precisely, we answer to the following question: when the discrete mode of a SLS is not generically observable, where can we place efficiently additional sensors to recover this property?

All the proposed results are based on classical combinatorial algorithms with polynomial order complexity. This may be an important criterion when we deal with large scale systems. Moreover, since we consider structured systems, our approach can be used during a conception stage.

The paper is organised as follows: after Section 2, which is devoted to the problem formulation, some definitions related to the graph-theoretic approach are given in Section 3. The main results are enounced in Section 4 before a brief conclusion.

\section{PROBLEM STATEMENT}

Consider the following SLS

$$
\Sigma:\left\{\begin{array}{l}
\dot{x}(t)=A\left(r_{t}\right) x(t)+B\left(r_{t}\right) u(t) \\
y(t)=C\left(r_{t}\right) x(t)+D\left(r_{t}\right) u(t)
\end{array}\right.
$$

where $x \in \mathbb{R}^{n}, u \in \mathbb{R}^{m}$ and $y \in \mathbb{R}^{p}$ are respectively the state vector, the unknown input vector and the output (measurement) vector and where $A(\cdot), B(\cdot), C(\cdot)$ and $D(\cdot)$ are real matrices of compatible dimensions. The exogenous and unobserved discrete mode variable (or switching signal) $r:[0, \infty) \rightarrow Q \stackrel{\text { def }}{=}\{1, \ldots, N\}$, is assumed, as in [3], to be right-continuous and only a finite number of jumps can occur in any finite interval of $[0, \infty)$. It results that all the system 
trajectories are well defined and infinitely right-differentiable over $[0, \infty)$. Zeno behaviors can thus not occur, even though no minimum dwell time is imposed. Note that, contrary to non-hybrid linear systems, when we study observability, we cannot remove the known inputs from the model, as discrete mode observability depends also on input-output links [8]. Nevertheless, without loss of generality and for the sake of homogeneity, all the inputs are assumed to be unknown. In fact, the control input signals, whose values are known, are considered to be measured i.e. we associate a virtual output equation of the form $y_{k^{\prime}}=u_{k}$ to these inputs. Moreover, our aim is to address, in the same framework autonomous SLS and SLS with known or unknown inputs. It results that, the discrete mode observability as it is defined in [7], cannot be used since it is dependent on input vector and so cannot be satisfied for autonomous SLS. We choose here to release this constraint on discrete mode observability to have a more general framework by imposing mode distinguishability not for all but for generic initial conditions $x_{0}$ and unknown inputs $u$ :

Definition 1. (Mode distinguishability) Two modes $q \in Q$ and $q^{\prime} \in Q$ with $\left(q \neq q^{\prime}\right)$ are distinguishable if at least one of the two following conditions holds:

- there exist an integer $s \geq 0$ and an expression $\Psi_{q}\left(y, \dot{y}, \ldots, y^{(s)}\right)=0$ which is satisfied for mode $q$ but is not satisfied for mode $q^{\prime}$ for almost all initial conditions $x_{0}$ and inputs $u$.

- there exist an integer $s^{\prime} \geq 0$ and an expression $\Psi_{q^{\prime}}\left(y, \dot{y}, \ldots, y^{\left(s^{\prime}\right)}\right)=0$ which is satisfied for mode $q^{\prime}$ but is not satisfied for mode $q$ for almost all initial conditions $x_{0}$ and inputs $u$.

Here, "for almost all initial conditions $x_{0}$ and inputs $u(t)$ " is to be understood as " for all $\left(x_{0}^{T}, u^{T}\right)^{T} \in \mathbb{R}^{n+m}$ except for the zero set of some polynomials with real coefficients in the $n+m$ initial state and input components. These polynomials can be written down explicitly, i.e. we can precisely describe when the mode distinguishability fails to be true. Obviously $u(t) \equiv 0$ and $x_{0}=0$ are two of these polynomials. The zero set of some polynomial forms a proper algebraic variety of $\mathbb{R}^{n+m}$ which has Lebesgue measure zero. So, by the expression "for almost all initial conditions $x_{0}$ and input functions $u$ " we mean for all initial conditions and inputs except the ones belonging to a proper algebraic variety in the state and input space.

The interpretation of Definition 1 is that $q$ is distinguishable from $q^{\prime}$ if, for generic initial states $x_{0}$ and unknown input $u$, we can rule out $q$ or $q^{\prime}$ when observing the output over $[0, T]$. Relatively to the definitions of [3], our notion of distinguishability of $q$ and $q^{\prime}$ is equivalent to the fact that $q$ is discernible from $q^{\prime}$ or vice-versa. The mutual mode discernibility, which is a dissymmetric property in [3], is equivalent to have both conditions of Definition 1 satisfied.

Definition 2. (Location observability) SLS $(\Sigma)$ is location observable if its modes are all distinguishable two-by-two i.e. $\forall q \in Q, \forall q^{\prime} \in Q$, with $q \neq q^{\prime}, q$ and $q^{\prime}$ are distinguishable.

Comparatively with the notion of location observability defined in $[8,7]$, our definition concerns as well as autonomous and non-autonomous systems. In [8,7], location observability is defined as the ability to reconstruct the mode starting from the knowledge of the input and the output, for any nonzero input value and for all initial conditions. Since we deal with unknown input systems, this definition is not applicable and it cannot be achieved for autonomous systems. In our definition, we relax this by accepting that the reconstruction of the mode may be possible not for all but for almost all inputs and initial condition values.

Location observability analysis can be reduced to the study of the distinguishability of each pair of modes. Thus, for the sake of simplicity, there is no loss of generality in considering in the first part of the paper, that we have only two modes. At the end of Section 4, we extend the obtained results to the multiple modes case.

Since we study structural properties, it is pertinent to deal with structured systems, for which we assume that only the sparsity pattern of matrices $A(q), B(q), C(q)$ and $D(q)$ is known for $q \in\{1,2\}$. So, to each entry of these matrices, we only know whether its value is fixed to zero, or that it has an unknown real value represented by a real parameter $\lambda_{i}$. The vector of these parameters is $\Lambda=\left(\lambda_{1}, \lambda_{2}, \ldots, \lambda_{h}\right)^{T}$ and it is assumed that $\Lambda$ can take any value in $\mathbb{R}^{h}$ or equivalently that parameters $\lambda_{i}$ are free. We denote by $A^{\lambda}(q), B^{\lambda}(q), C^{\lambda}(q)$ and $D^{\lambda}(q)$ respectively the matrices obtained by replacing the nonzeros in $A(q), B(q), C(q)$ and $D(q)$, for $q \in\{1,2\}$ by the corresponding parameters $\lambda_{i}$ and we denote

$$
\Sigma_{\Lambda}:\left\{\begin{array}{l}
\dot{x}(t)=A^{\lambda}\left(r_{t}\right) x(t)+B^{\lambda}\left(r_{t}\right) u(t) \\
y(t)=C^{\lambda}\left(r_{t}\right) x(t)+D^{\lambda}\left(r_{t}\right) u(t)
\end{array}\right.
$$

If all parameters $\lambda_{i}$ are numerically fixed, we obtain a so-called admissible realization of SSLS $\left(\Sigma_{\Lambda}\right)$. We say that a property is true generically for SSLS $\left(\Sigma_{\Lambda}\right)$ if it is true for almost its realizations or equivalently for almost all parameters $\lambda_{i}$.

Location observability analysis is based on the distinguishability of the structured models associated to the two discrete modes. So, it is pertinent and necessary to highlight the similarities and the differences between the models associated to these modes. Thus, we decompose each structured matrix into two parts: the first one is common to the two modes and the second one is specific to each mode i.e. for $q \in\{1,2\}, A^{\lambda}(q)=A_{0}^{\lambda}+$ $A_{q}^{\lambda}, B^{\lambda}(q)=B_{0}^{\lambda}+B_{q}^{\lambda}, C^{\lambda}(q)=C_{0}^{\lambda}+C_{q}^{\lambda}$ and $D^{\lambda}(q)=D_{0}^{\lambda}+$ $D_{q}^{\lambda}$. When we write the matrices under this form, we assume that the entries of all these matrices are free. These notations are generalized to the multi-mode case in subsection 4.5. To summarize, our first aim is to provide sufficient conditions to check if a SSLS is generically location observable i.e. is location observable for almost all its realizations or equivalently for almost all parameters $\lambda_{i}$. Next, when these conditions are not satisfied, we provide additional sensors' locations which allow to recover the location observability property.

\section{GRAPHICAL REPRESENTATION OF STRUCTURED SWITCHING LINEAR SYSTEMS}

The digraph associated to $\left(\Sigma_{\Lambda}\right)$ is noted $\mathcal{G}\left(\Sigma_{\Lambda}\right)$. It is constituted by a vertex set $\mathcal{V}$ and an edge set $\mathcal{E}$ i.e. $\mathcal{G}\left(\Sigma_{\Lambda}\right)=(\mathcal{V}, \mathcal{E})$. The vertices are associated to the continuous state, the input and the output components of $\left(\Sigma_{\Lambda}\right)$ and the directed edges represent links between these variables. More precisely, $\mathcal{V}=\mathbf{X} \cup \mathbf{U} \cup \mathbf{Y}$, where $\mathbf{X}=\left\{\mathbf{x}_{\mathbf{1}}, \ldots, \mathbf{x}_{\mathbf{n}}\right\}, \mathbf{U}=\left\{\mathbf{u}_{\mathbf{1}}, \ldots, \mathbf{u}_{\mathbf{m}}\right\}$ and $\mathbf{Y}=\left\{\mathbf{y}_{\mathbf{1}}, \ldots, \mathbf{y}_{\mathbf{p}}\right\}$ are respectively the set of state, input and output vertices. For $q \in\{0,1,2\}$, we define $\mathcal{E}_{q}=A_{q}$-edges $\cup B_{q}$-edges $\cup C_{q}$-edges $\cup D_{q}$-edges, where, for $q \in\{0,1,2\}, A_{q}$-edges $=\left\{\left(\mathbf{x}_{\mathbf{j}}, \mathbf{x}_{\mathbf{i}}\right) \mid A_{q}(i, j) \neq 0\right\}$, $B_{q}$-edges $=\left\{\left(\mathbf{u}_{\mathbf{j}}, \mathbf{x}_{\mathbf{i}}\right) \mid B_{q}(i, j) \neq 0\right\}, C_{q}$-edges $=$ $\left\{\left(\mathbf{x}_{\mathbf{j}}, \mathbf{y}_{\mathbf{i}}\right) \mid C_{q}(i, j) \neq 0\right\} \quad$ and $D_{q}$-edges $=$ $\left\{\left(\mathbf{u}_{\mathbf{j}}, \mathbf{y}_{\mathbf{i}}\right) \mid D_{q}(i, j) \neq 0\right\}$. Finally the edge set is $\mathcal{E}=\bigcup_{q=0}^{2} \mathcal{E}_{q}$.

Here, $M(i, j)$ is the $(i, j)$ th element of matrix $M$ and $\left(\mathbf{v}_{\mathbf{1}}, \mathbf{v}_{\mathbf{2}}\right)$ denotes a directed edge from vertex $\mathbf{v}_{\mathbf{1}} \in \mathcal{V}$ to vertex $\mathbf{v}_{\mathbf{2}} \in \mathcal{V}$. The edges included in $\mathcal{E}_{0}$ represent the common part of the two modes' models, while $\mathcal{E}_{q}$, for $q \in\{1,2\}$ is related to the specific part of each mode's model. For the sake of clarity, the 
vertices are written in bold fonts to differentiate them from the corresponding variables. Each edge is associated to a free non-zero parameter of the system's model called the weight of the edge. Note that number $q$ is written under each $\mathcal{E}_{q}$-edge and represents its index.

Example 1. To the system defined by the following matrices, we associate the digraph in Figure 1.

$A_{0}^{\lambda}=\left(\begin{array}{cccccccc}0 & 0 & 0 & 0 & 0 & 0 & 0 & 0 \\ 0 & 0 & \lambda_{1} & 0 & 0 & 0 & 0 & 0 \\ 0 & \lambda_{2} & 0 & 0 & 0 & 0 & 0 & 0 \\ 0 & 0 & 0 & 0 & \lambda_{3} & 0 & 0 & \lambda_{4} \\ 0 & \lambda_{5} & 0 & 0 & 0 & 0 & 0 & 0 \\ 0 & 0 & 0 & 0 & 0 & 0 & 0 & 0 \\ 0 & 0 & 0 & 0 & \lambda_{6} & \lambda_{7} & 0 & 0 \\ 0 & 0 & 0 & 0 & 0 & 0 & \lambda_{8} & 0\end{array}\right), B_{0}^{\lambda}=\left(\begin{array}{c}0 \\ 0 \\ 0 \\ 0 \\ 0 \\ \lambda_{12} \\ 0 \\ 0\end{array}\right), C_{0}^{\lambda}=$ $\left(\begin{array}{cccccccc}\lambda_{13} & 0 & 0 & 0 & 0 & 0 & 0 & 0 \\ 0 & 0 & 0 & 0 & \lambda_{14} & 0 & 0 & 0 \\ 0 & 0 & 0 & \lambda_{15} & 0 & 0 & 0 & 0\end{array}\right)$ $\left(\begin{array}{cccccccc}\lambda_{1} & 0 & 0 & 0 & \lambda_{14} & 0 & 0 & 0 \\ 0 & 0 & 0 & \lambda_{15} & 0 & 0 & 0 & 0 \\ 0 & 0 & 0 & 0 & 0 & 0 & 0 & \lambda_{16}\end{array}\right)$, all the entries of $A_{1}^{\lambda}$ are zero except $A_{1}^{\lambda}(1,2)=\lambda_{9}$, all the entries of $A_{2}^{\lambda}$ are zero except $A_{2}^{\lambda}(5,2)=\lambda_{10}$ and $A_{2}^{\lambda}(7,6)=\lambda_{11}$, the elements of matrices $B_{1}^{\lambda}, B_{2}^{\lambda}, C_{1}^{\lambda}, C_{2}^{\lambda}, D_{0}^{\lambda}, D_{1}^{\lambda}$ and $D_{2}^{\lambda}$ are equal to zero.

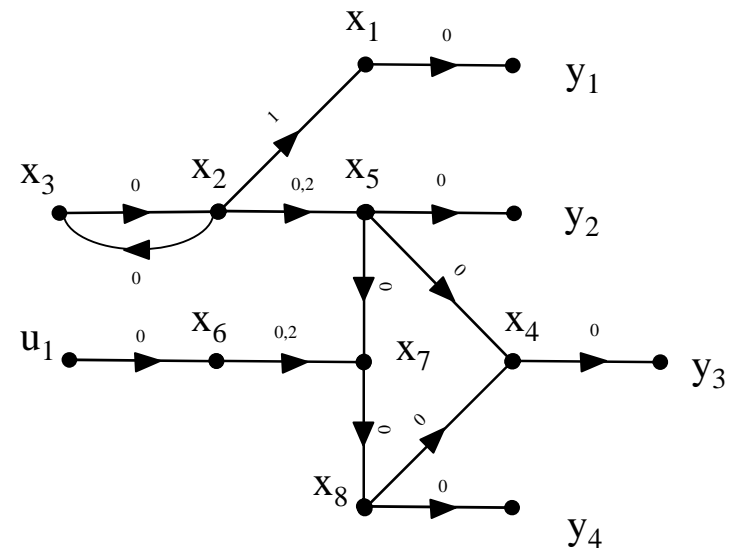

Figure 1. Digraph associated to system of Example 1

The digraph representing the SSLS is built from the superposition of the digraphs related to each mode. In order to study the properties of the system associated to a specific mode $q$, we have to restrict the edge set to $\mathcal{E}_{0} \cup \mathcal{E}_{q}$. In this context, many of the functions and specific vertex subsets, defined below, present an index $q$ related to the considered mode.

Let us now give some useful definitions and notations.

- A path $P$ is denoted $P=\mathbf{v}_{\mathbf{s}_{\mathbf{o}}} \rightarrow \mathbf{v}_{\mathbf{s}_{1}} \rightarrow \ldots \rightarrow \mathbf{v}_{\mathbf{s}_{\mathbf{i}}}$, where $\left(\mathbf{v}_{\mathbf{s}_{\mathbf{j}}}, \mathbf{v}_{\mathbf{s}_{\mathbf{j}+1}}\right) \in \mathcal{E}$ for $j=0,1, \ldots, i-1$. We say in this case that $P$ covers $\mathbf{v}_{\mathbf{s}_{0}}, \mathbf{v}_{\mathbf{s}_{1}}, \ldots, \mathbf{v}_{\mathbf{s}_{\mathrm{i}}}$.

- The weight of $P$ is the product of the weights of all its edges. - A path is simple when every vertex occurs only once in this path.

- A cycle is a path of the form $\mathbf{v}_{\mathbf{s}_{0}} \rightarrow \mathbf{v}_{\mathbf{s}_{1}} \rightarrow \ldots \rightarrow \mathbf{v}_{\mathbf{s}_{\mathbf{i}}} \rightarrow$ $\mathbf{v}_{\mathbf{s}_{0}}$, where $\mathbf{v}_{\mathbf{s}_{\mathbf{0}}}, \mathbf{v}_{\mathbf{s}_{1}}, \ldots, \mathbf{v}_{\mathbf{s}_{\mathbf{i}}}$ are distinct.

- For $q \in\{1,2\}$, we say that path $P$ is included in $\mathcal{E}_{0} \cup \mathcal{E}_{q}$ if all its edges are included in $\mathcal{E}_{0} \cup \mathcal{E}_{q}$.

- Some paths (resp. cycles) are disjoint if they have no common vertex.

- $P$ is a $\mathbf{Y}$-topped path if its end vertex belongs to $\mathbf{Y}$. A $\mathbf{Y}$ topped path family consists of disjoint simple $\mathbf{Y}$-topped paths. In the sequel, $\mathcal{V}_{1}$ and $\mathcal{V}_{2}$ represent two subsets of $\mathcal{V}$. We denote by $\operatorname{card}(\cdot)$ the cardinality function and $\mathcal{V}_{1} \backslash \mathcal{V}_{2}$ is the set of elements in $\mathcal{V}_{1}$ which are not in $\mathcal{V}_{2}$.

- A path $P=\mathbf{v}_{\mathbf{s}_{0}} \rightarrow \mathbf{v}_{\mathbf{s}_{1}} \rightarrow \ldots \rightarrow \mathbf{v}_{\mathbf{s}_{\mathbf{i}}}$ is said a $\mathcal{V}_{1}-\mathcal{V}_{2}$ path if $\mathbf{v}_{\mathbf{s}_{\mathbf{o}}} \in \mathcal{V}_{1}$ and $\mathbf{v}_{\mathbf{s}_{\mathbf{i}}} \in \mathcal{V}_{2}$. Moreover, if the only vertex of $P$ which belongs to $\mathcal{V}_{1}$ is $\mathbf{v}_{\mathbf{s}_{0}}$ and the only vertex of $P$ which belongs to $\mathcal{V}_{2}$ is $\mathbf{v}_{\mathbf{s}_{\mathbf{i}}}, P$ is called a direct $\mathcal{V}_{1}-\mathcal{V}_{2}$ path.

- For $q=\{1,2\}, \rho_{q}\left[\mathcal{V}_{1}, \mathcal{V}_{2}\right]$ is the maximal number of disjoint $\mathcal{V}_{1}-\mathcal{V}_{2}$ paths included in $\mathcal{E}_{0} \cup \mathcal{E}_{q}$. Moreover, a set of $\rho_{q}\left[\mathcal{V}_{1}, \mathcal{V}_{2}\right]$ disjoint $\mathcal{V}_{1}-\mathcal{V}_{2}$ paths included in $\mathcal{E}_{0} \cup \mathcal{E}_{q}$ is a maximum $\mathcal{V}_{1}-\mathcal{V}_{2}$ linking in $\mathcal{E}_{0} \cup \mathcal{E}_{q}$.

- For $q \in\{1,2\}, V_{e s s, q}\left[\mathcal{V}_{1}, \mathcal{V}_{2}\right]$ is the vertex subset including the vertices present in all the maximum $\mathcal{V}_{1}-\mathcal{V}_{2}$ linkings included in $\mathcal{E}_{0} \cup \mathcal{E}_{q}$.

- For $q \in\{1,2\}$, there exists a unique vertex subset noted $S_{q}^{o}\left[\mathcal{V}_{1}, \mathcal{V}_{2}\right]$ and called minimum output separator which is the set of begin vertices of all direct $V_{e s s, q}\left[\mathcal{V}_{1}, \mathcal{V}_{2}\right]-\mathcal{V}_{2}$ paths included in $\mathcal{E}_{0} \cup \mathcal{E}_{q}$.

In Example $1, \rho_{1}[\mathbf{U}, \mathbf{Y}]=\rho_{2}[\mathbf{U}, \mathbf{Y}]=1$ and $\rho_{1}\left[\left\{\mathbf{x}_{1}, \mathbf{x}_{\mathbf{2}}, \mathbf{x}_{\mathbf{3}}\right\}, \mathbf{Y}\right]=\rho_{2}\left[\left\{\mathbf{x}_{1}, \mathbf{x}_{\mathbf{2}}, \mathbf{x}_{\mathbf{3}}\right\}, \mathbf{Y}\right]=2$. Moreover, $V_{e s s, 2}\left[\left\{\mathbf{x}_{\mathbf{3}}\right\}, \mathbf{Y}\right]=\left\{\mathbf{x}_{\mathbf{3}}, \mathbf{x}_{\mathbf{2}}, \mathbf{x}_{\mathbf{5}}\right\}$ but $V_{e s s, 1}\left[\left\{\mathbf{x}_{\mathbf{3}}\right\}, \mathbf{Y}\right]=$ $\left\{\mathbf{x}_{3}, \mathbf{x}_{2}\right\}$ because there exists in $\mathcal{E}_{0} \cup \mathcal{E}_{1}$ a path which from $\mathrm{x}_{3}$ to $\mathrm{Y}$ which does not cover $\mathrm{x}_{5}: \mathrm{x}_{3} \rightarrow \mathrm{x}_{2} \rightarrow \mathrm{x}_{1} \rightarrow$ $\mathbf{y}_{\mathbf{1}}$. Finally, $S_{1}^{o}[\mathbf{U}, \mathbf{Y}]=\left\{\mathbf{x}_{\mathbf{8}}\right\}, S_{2}^{o}\left[\left\{\mathbf{x}_{\mathbf{3}}\right\}, \mathbf{Y}\right]=\left\{\mathbf{x}_{\mathbf{5}}\right\}$ and $S_{1}^{o}\left[\left\{\mathbf{x}_{\mathbf{3}}\right\}, \mathbf{Y}\right]=\left\{\mathbf{x}_{\mathbf{2}}\right\}$.

Function $\rho_{q}$ and the two vertex subsets $V_{e s s, q}$ and $S_{q}^{o}$ constitute the classical graphic-notions used in most of the results related to the structural analysis using a graph-theoretic approach. Indeed, $\rho_{q}$ is associated to the generic rank of transfer matrices and pencil matrices for example and is useful in the analysis of many problems as disturbance-rejection, input-output decoupling, fault detection and isolation [9]. Subsets $V_{e s s, q}$ and $S_{q}^{o}$ are often used, as we will see later, in the system's decomposition [17] or in the graph simplification because for some properties, analysing all the graph is not always necessary.

- In $\mathcal{G}\left(\Sigma_{\Lambda}\right)$, for each mode $q$, two vertices $\mathbf{v}_{\mathbf{i}}$ and $\mathbf{v}_{\mathbf{j}}$ are said to be strongly connected in mode $q$, if it exists a path included in $\mathcal{E}_{0} \cup \mathcal{E}_{q}$ from $\mathbf{v}_{\mathbf{i}}$ to $\mathbf{v}_{\mathbf{j}}$ and a path included in $\mathcal{E}_{0} \cup \mathcal{E}_{q}$ from $\mathbf{v}_{\mathbf{j}}$ to $\mathbf{v}_{\mathbf{i}}$. In each mode, the relation "is strongly connected to" is an equivalence relation and we can define its equivalence classes. We call each equivalent class related in mode $q$ by a $q$-strongly connected component $(q$-scc). These $q$-scc are well known in the graph theory [12]. They can be ordered using a partial order relation " $\preccurlyeq$ " defined between two $q$-scc $\mathbf{V}_{\mathbf{i}}$ and $\mathbf{V}_{\mathbf{j}}$ as $\mathbf{V}_{\mathbf{i}} \preccurlyeq \mathbf{V}_{\mathbf{j}}$ if there exists a path, included in $\mathcal{E}_{0} \cup \mathcal{E}_{q}$, from an element of $\mathbf{V}_{\mathbf{j}}$ to an element of $\mathbf{V}_{\mathbf{i}}$.

- For each mode $q$, a $q$-scc $\mathbf{V}_{\mathbf{i}}$ is maximal if there is no distinct $q$-sec $\mathbf{V}_{\mathbf{j}}$ such that $\mathbf{V}_{\mathbf{i}} \preccurlyeq \mathbf{V}_{\mathbf{j}}$.

- We define, for each mode $q$, the union of all maximal $q$-scc:

$$
\begin{aligned}
\mathbf{V}_{\text {or }, \mathbf{q}} & =\left\{\mathbf{v} \in \mathbf{V}_{\mathbf{i}} \text {, where } \mathbf{V}_{\mathbf{i}} \text { is a maximal } q \text {-scc }\right\} \\
& =\bigcup_{\mathbf{V}_{\mathbf{i}} \text { is a maximal } q \text {-scc }} \mathbf{V}_{\mathbf{i}}
\end{aligned}
$$

. In Example 1, the 1-scc are: $\left\{\mathbf{u}_{1}\right\},\left\{\mathbf{x}_{1}\right\},\left\{\mathbf{x}_{\mathbf{2}}, \mathbf{x}_{\mathbf{3}}\right\},\left\{\mathbf{x}_{\mathbf{4}}\right\}$, $\left\{\mathbf{x}_{5}\right\},\left\{\mathbf{x}_{6}\right\},\left\{\mathbf{x}_{7}\right\},\left\{\mathbf{x}_{8}\right\},\left\{\mathbf{y}_{1}\right\},\left\{\mathbf{y}_{2}\right\},\left\{\mathbf{y}_{3}\right\},\left\{\mathbf{y}_{4}\right\}$. The maximal ones are $\left\{\mathbf{u}_{\mathbf{1}}\right\}$ and $\left\{\mathbf{x}_{\mathbf{2}}, \mathbf{x}_{\mathbf{3}}\right\}$ and so $\mathbf{V}_{\mathbf{o r}, \mathbf{1}}=$ $\left\{\mathbf{u}_{\mathbf{1}}, \mathbf{x}_{\mathbf{2}}, \mathbf{x}_{\mathbf{3}}\right\}$. Furthermore, $S_{1}^{o}\left[\mathbf{V}_{\mathrm{or}, \mathbf{1}}, \mathbf{Y}\right]=\left\{\mathbf{x}_{\mathbf{2}}, \mathbf{x}_{\mathbf{8}}\right\}$. In mode 2 , the strongly connected components are the same. However, since there is no edge between $\mathbf{x}_{2}$ and $\mathbf{x}_{1}$, the latter is also a maximal 2-scc. Therefore, $\mathbf{V}_{\text {or, } 2}=\left\{\mathbf{u}_{1}, \mathbf{x}_{1}, \mathbf{x}_{2}, \mathbf{x}_{3}\right\}$. Furthermore, $S_{2}^{o}\left[\mathbf{V}_{\mathbf{o r}, 2}, \mathbf{Y}\right]=\left\{\mathbf{y}_{1}, \mathbf{x}_{\mathbf{5}}, \mathbf{x}_{\mathbf{8}}\right\}$.

\section{MAIN RESULTS}

\subsection{Preliminaries}

In a first stage, hereafter we recall some existing results useful to establish our observability conditions. These results are 
mainly issued from $[12,14,17,18,9]$.

Consider subset $\mathbf{W}_{\mathbf{q}}=S_{q}^{o}\left[\mathbf{V}_{\mathbf{o r}, \mathbf{q}}, \mathbf{Y}\right]$. We have, according to Menger's Theorem, that $\operatorname{card}\left(\mathbf{W}_{\mathbf{q}}\right)=\rho_{q}\left[\mathbf{V}_{\text {or, } \mathbf{q}}, \mathbf{Y}\right]$. Furthermore, let us denote by $\overline{\mathbf{X}}_{\mathbf{q}}$ all the state vertices belonging to direct $\mathbf{W}_{\mathbf{q}}-\mathbf{Y}$ paths and which are not in $\mathbf{W}_{\mathbf{q}}$. The dynamics of the state components associated to $\overline{\mathbf{X}}_{\mathbf{q}}$ is then on the form :

$$
\bar{\Sigma}_{q}:\left\{\begin{aligned}
\dot{\bar{x}}_{q} & =\bar{A}_{q}^{\lambda} \bar{x}_{q}+\bar{B}_{q}^{\lambda} w_{q} \\
y & =\bar{C}_{q}^{\lambda} \bar{x}_{q}+\bar{D}_{q}^{\lambda} w_{q}
\end{aligned}\right.
$$

where $w_{q}, \bar{x}_{q}$ are the input and state components associated respectively to vertices of $\bar{W}_{q}$ and $\bar{X}_{q}$.

Furthermore, since $\mathbf{W}_{\mathbf{q}}=S_{q}^{o}\left[\mathbf{V}_{\mathbf{o r}, \mathbf{q}}, \mathbf{Y}\right]$, an important remark can be done now and is summarized in the following lemma:

Lemma 1. Considering subsystem $\left(\bar{\Sigma}_{q}\right)$ constituted by input $w_{q}$, state $\bar{x}_{q}$ and output $y$. For each $\mathbf{Y}_{\mathbf{u}}=$ $\left\{\mathbf{y}_{\mathbf{i}_{1}}, \mathbf{y}_{\mathbf{i}_{2}}, \ldots, \mathbf{y}_{\mathbf{i}_{\mathbf{k}}}\right\} \subset \mathbf{Y}$ such that $\rho_{q}\left[\mathbf{W}_{\mathbf{q}}, \mathbf{Y}_{\mathbf{u}}\right]=$ $\operatorname{card}\left(\mathbf{W}_{\mathbf{q}}\right)$, there exist, generically, a matrix $G$, a function $\varphi$ and an integer $\nu \leq n_{q} \stackrel{\text { def }}{=} \operatorname{card}\left(\mathbf{X}_{\mathbf{q}}\right)$ such that $w_{q}=\varphi\left(Y_{u}, \dot{Y}_{u}, \ldots, Y_{u}^{(\nu)}\right)+G \bar{x}_{q}$, where $Y_{u}^{(\nu)}=$ $\left(y_{i_{1}}^{(\nu)}, y_{i_{2}}^{(\nu)}, \ldots, y_{i_{k}}^{(\nu)}\right)^{T}$

Proof: According to [9] (Theorem 4), for subsystem $\left(\bar{\Sigma}_{q}\right)$, the existence of $\mathbf{Y}_{\mathbf{u}}=\left\{\mathbf{y}_{\mathbf{i}_{1}}, \mathbf{y}_{\mathbf{i}_{2}}, \ldots, \mathbf{y}_{\mathbf{i}_{\mathbf{k}}}\right\} \subset \mathbf{Y}$ such that $\rho_{q}\left[\mathbf{W}_{\mathbf{q}}, \mathbf{Y}_{\mathbf{u}}\right]=\operatorname{card}\left(\mathbf{W}_{\mathbf{q}}\right)$, implies the invertibility of $\left(\bar{\Sigma}_{q}\right)$ using only $Y_{u}$. Thus, using the inversion algorithm of [15], we can express the input of a such system i.e. $w_{q}$ in function of its state $\bar{x}_{q}$ and its output components $Y_{u}$ and their derivatives. Thus, we have that there exist, generically, a matrix $G$, a function $\varphi$ and an integer $\nu \leq n_{q}$ such that $w_{q}=\varphi\left(Y_{u}, \dot{Y}_{u}, \ldots, Y_{u}^{(\nu)}\right)+G \bar{x}_{q}$, where $Y_{u}^{(\nu)}=\left(y_{i_{1}}^{(\nu)}, y_{i_{2}}^{(\nu)}, \ldots, y_{i_{k}}^{(\nu)}\right)^{T}$.

Substituting this in Equations (3) for mode $q$, we have:

$$
\left\{\begin{aligned}
\dot{\bar{x}}_{q} & =\left(\bar{A}_{q}^{\lambda}+\bar{B}_{q}^{\lambda} G\right) \bar{x}_{q}+\bar{B}_{q}^{\lambda} \varphi\left(Y_{u}, \dot{Y}_{u}, \ldots, Y_{u}^{(\nu)}\right) \\
& \stackrel{\text { def }}{=} \\
A & \bar{x}_{q}+\varphi_{x}\left(Y_{u}, \dot{Y}_{u}, \ldots, Y_{u}^{(\nu)}\right) \\
y & =\left(\bar{C}_{q}^{\lambda} \bar{x}_{q}+\bar{D}_{q}^{\lambda} G\right) \bar{x}_{q}+\bar{D}_{q}^{\lambda} \varphi\left(Y_{u}, \dot{Y}_{u}, \ldots, Y_{u}^{(\nu)}\right) \\
& \stackrel{\text { def }}{=} \tilde{C} \bar{x}_{q}+\varphi_{y}\left(Y_{u}, \dot{Y}_{u}, \ldots, Y_{u}^{(\nu)}\right)
\end{aligned}\right.
$$

Remark: To avoid heavy notations, even if function $\varphi$ and matrices $G, \tilde{A}$ and $\tilde{C}$ depend on parameters $\lambda_{i}$ and on mode $q$, we omit to write superscript $\lambda$ and subscript $q$ on each of them.

\subsection{Location observability analysis}

Using the previous settings and definitions, we enounce the first result of the paper. It characterizes a sufficient condition for the existence of an algebraic equation depending on a specific mode to ensure mode distinguishability.

Proposition 1. SSLS $\left(\Sigma_{\Lambda}\right)$, with two possible modes $q \in$ $\{1,2\}$, associated to digraph $\mathcal{G}\left(\Sigma_{\Lambda}\right)$ is generically location observable if for some $q \in\{1,2\}$, there exists a direct $S_{q}^{o}\left[\mathbf{V}_{\text {or, }}, \mathbf{Y}\right]-\mathbf{Y}$ path included in $\mathcal{E}_{0} \cup \mathcal{E}_{q}$ and containing at least one edge of $\mathcal{E}_{q}$.

\section{Proof:}

Consider that this condition is satisfied and let denote by $\mathbf{v}_{\mathbf{i}}$ the vertex of $S_{q}^{o}\left[\mathbf{V}_{\mathbf{o r}, \mathbf{q}}, \mathbf{Y}\right]$ from which there is a direct $S_{q}^{o}\left[\mathbf{V}_{\text {or }, \mathbf{q}}, \mathbf{Y}\right]-\mathbf{Y}$ path $P$ included in $\mathcal{E}_{0} \cup \mathcal{E}_{q}$ and containing at least an edge $e_{\kappa}$ of $\mathcal{E}_{q}$. Let us denote by $\lambda_{\kappa}$ the non-zero parameter (or weight) associated to $e_{\kappa}$. Let $\mathbf{y}_{\mathbf{j}}$ be the end of $P$ and $\ell$ its length. Since $\mathbf{v}_{\mathbf{i}}$ belongs to $S_{q}^{o}\left[\mathbf{V}_{\mathbf{o r}, \mathbf{q}}, \mathbf{Y}\right]$, it exists a subset $\mathbf{Y}_{\text {or }}=\left\{\mathbf{y}_{\mathbf{i}_{1}}, \mathbf{y}_{\mathbf{i}_{2}}, \ldots, \mathbf{y}_{\mathbf{i}_{\mathbf{k}}}\right\} \subset \mathbf{Y} \backslash\left\{\mathbf{y}_{\mathbf{j}}\right\}$ such that $\rho_{q}\left[S^{o}\left[\mathbf{V}_{\mathbf{o r}, \mathbf{q}}, \mathbf{Y}\right], \mathbf{Y}_{\mathbf{~ o r}}\right]=\operatorname{card}\left(\mathbf{S}_{\mathbf{q}}^{\mathbf{o}}\left[\mathbf{V}_{\mathbf{o r}, \mathbf{q}}, \mathbf{Y}\right]\right)$ without using edges and vertices of $P$. Thus, as it is shown in Lemma 1 , there exist a matrix $G$, a function $\varphi$ and an integer $\nu \leq n_{q}$ such that the dynamics equation of subsystem $\left(\bar{\Sigma}_{q}\right)$ is in the form (4). Since the elements of $G$ are represented by edges belonging to the $\mathbf{Y}_{\text {or-topped }}$ paths, they are independent from the elements of $\tilde{A}$. From the characteristic equation of matrix $\tilde{A}$, we can write an equation as

$$
\left(\widetilde{C}_{j} \tilde{A}^{n_{1}}+\ldots+a_{k} \tilde{C}_{j} \tilde{A}^{k}+\ldots+a_{0} \tilde{C}_{j} \tilde{A}\right) X=0
$$

where also $\tilde{C}_{j}$ is the line of matrix $\widetilde{C}$ related to output $y_{j}$ in mode $q$ in (4) i.e. $y_{j}=\tilde{C}_{j} X+\varphi_{y, j}\left(Y_{o r}, \dot{Y}_{o r}, \ldots, Y_{o r}^{(\nu)}\right)$ and so, $\tilde{C}_{j} \tilde{A}_{1,1}^{k} X=y_{j}^{(k)}-\tilde{C}_{j}\left(\varphi_{x}^{(k-1)}\left(Y_{\text {or }}, \dot{Y}_{\text {or }}, \ldots, Y_{\text {or }}{ }^{(\nu)}\right)+\right.$ $\tilde{A}_{1,1} \varphi_{x}^{(k-2)}\left(Y_{\text {or }}, \dot{Y}_{\text {or }}, \ldots, Y_{\text {or }}{ }^{(\nu)}\right)+\ldots+$ $\left.\tilde{A}_{1,1}^{k-2} \dot{\varphi}_{x}\left(Y_{\text {or }}, \dot{Y}_{\text {or }}, \ldots, Y_{\text {or }}{ }^{(\nu)}\right)\right)-\varphi_{y, j}^{(k)}\left(Y_{\text {or }}, \dot{Y}_{\text {or }}, \ldots, Y_{\text {or }}^{(\nu)}\right)$. Since there exists a path of length $\ell$ from $\mathbf{v}_{\mathbf{i}}$ to $\mathbf{y}_{\mathbf{j}}$ containing an edge $e_{\kappa}$, term $C_{j} \tilde{A}_{1,1}^{\ell-1} \varphi_{x}^{(k-\ell)}\left(Y_{o r}, \dot{Y}_{o r}, \ldots, Y_{o r}{ }^{(\nu)}\right)$, for all $k \geq \ell$, is not zero and depends on the element of $A_{q}$ denoted previously $\lambda_{\kappa}$ which is specific to location $q$. In particular, first term $\tilde{C}_{j} \tilde{A}^{n_{1}}$, which is present in relation (5) is not zero and depends on $\lambda_{\kappa}$. Thus, equation (5) leads to an algebraic equation $\Psi_{q}\left(Y, \dot{Y}, \ldots, Y^{(s)}\right)=0$ parameterized by at least an element of $A_{q}$ and so satisfied only when mode $q$ occurs. So, the system is location observable.

Comments and interpretation: To establish location observability, we must first search in the graph the edges, called specific edges, which are specific to one mode. Next, we interest to the position of these edges w.r.t. the output vertices in order to analyse if it is possible to obtain an algebraic equation specific to a mode. If a specific edge belongs to any $S_{q}^{o}\left[\mathbf{V}_{\text {or, }}, \mathbf{Y}\right]-\mathbf{Y}$ path then the mode distinguishability is possible.

Note that the condition of Proposition 1 generalizes the condition $[7,8]$ of location observability for non autonomous systems, where the inputs are measured $(\exists i \geq 0$ such that $\left.C^{\lambda}(1)\left(A^{\lambda}\right)^{i}(1) B^{\lambda}(1) \neq C^{\lambda}(2)\left(A^{\lambda}\right)^{i}(2) B^{\lambda}(2)\right)$. Note that if there exists an equation characterising mode $q$, then the one characterizing mode $q^{\prime} \neq q$ cannot be satisfied by mode $q$. So, both the conditions of Definition 1 are satisfied and we can rule out mode $q^{\prime}$ when observing output related to mode $q$ and also mode $q$ when observing output related to mode $q^{\prime}$. Using definition of [3], this means, that the two modes $q$ and $q^{\prime}$ are mutually discernible.

Exemple 1 Let us see if it is possible to find a specific equation characterizing mode 1 . Recall that $S_{1}^{o}\left[\mathbf{V}_{\mathbf{o r}, \mathbf{1}}, \mathbf{Y}\right]=\left\{\mathbf{x}_{\mathbf{2}}, \mathbf{x}_{\mathbf{8}}\right\}$. Furthermore, there is one edge $\left(\mathbf{x}_{2}, \mathbf{x}_{1}\right)$ which is specific to mode 1 and which belongs to a $S_{1}^{o}\left[\mathbf{V}_{\mathbf{o r}, \mathbf{1}}, \mathbf{Y}\right]-\mathbf{Y}$ path. So, condition of Proposition 1 is satisfied and the system is generically location observable. Indeed, this graphic condition characterizes algebraic relation $\frac{\dot{y}_{1}}{\lambda_{9} \lambda_{13}}-\frac{\dot{y}_{2}}{\lambda_{5} \lambda_{14}}=0$ depending on $\lambda_{9}$ which is specific to mode 1 only. This relation is not satisfied for mode 2 because, in this case $\dot{y}_{1}$ is identically zero. Thus, relation $\dot{y}_{1}=0$ is specific to mode 2 even if there is no specific coefficient to mode 2 which appears.

\subsection{Additional sensor location to recover the mode distinguishability}

The aim of this part of the paper is to study additional sensor placement when the condition of Proposition 1 is not satisfied. 
In the sequel, we define a new output vector $\Upsilon$ representing the additional sensors which collect the new measurements $\Upsilon(t)=H_{x}^{\lambda} x(t)+H_{u}^{\lambda} u(t)$ independently from the mode, for practical consistency. Hence, we denote the completed system by $\Sigma_{\Lambda}^{c}$ :

$$
\Sigma_{\Lambda}^{c}:\left\{\begin{aligned}
\dot{x}(t) & =A^{\lambda}\left(r_{t}\right) x(t)+B^{\lambda}\left(r_{t}\right) u(t) \\
y(t) & =C^{\lambda}\left(r_{t}\right) x(t)+D^{\lambda}\left(r_{t}\right) u(t) \\
\Upsilon(t) & =H_{x}^{\lambda} x(t)+H_{u}^{\lambda} u(t)
\end{aligned}\right.
$$

The additional sensor components can be represented by vertex set $\Upsilon$ and edge subsets $H_{x}$-edges and $H_{u}$-edges from respectively $\mathbf{X}$ to $\boldsymbol{\Upsilon}$ and $\mathbf{U}$ to $\boldsymbol{\Upsilon}$.

Proposition 1. Consider SSLS $\left(\Sigma_{\Lambda}\right)$, with two

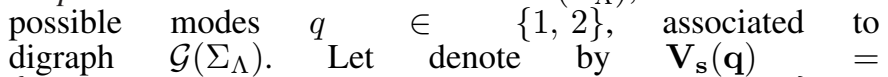
$\left\{\mathbf{v} \in \mathcal{V}, \mathbf{v}\right.$ is the begin vertex of an edge belonging to $\left.\mathcal{E}_{q}\right\}$. To recover the sufficient condition of Proposition $\bigcup_{q \in\{1,2\}}\left(V_{e s s, q}\left[\mathbf{V}_{\text {or }, \mathbf{q}}, \mathbf{Y}\right] \cap \quad\left\{\mathbf{v}_{\mathbf{i}}\right.\right.$ such that $\exists$ a simple $\mathbf{v}_{\mathbf{i}}-\mathbf{Y}$ path covering an element of $\left.\left.\mathbf{V}_{\mathbf{s}}(\mathbf{q})\right\}\right)$

Proof: Assume that we place an additional sensor on a vertex which is included in $\bigcup_{q \in\{1,2\}}\left(V_{\text {ess }, q}\left[\mathbf{V}_{\text {or, }}, \mathbf{Y}\right] \cap\left\{\mathbf{v}_{\mathbf{i}}\right.\right.$ such that $\exists$ a simple $\mathbf{v}_{\mathbf{i}}-\mathbf{Y}$ path covering an element of $\left.\left.\mathbf{V}_{\mathbf{s}}(\mathbf{q})\right\}\right)$.

Since this vertex denoted $\mathbf{v}_{\mathbf{i}}$ belongs to $V_{\text {ess }, q}\left[\mathbf{V}_{\text {or, }}, \mathbf{Y}\right]$ and since it will exist a new path from it to an output vertex (the additional sensor) then $\mathbf{v}_{\mathbf{i}}$ will be included in the output separator set of the extended system i.e. $\mathbf{v}_{\mathbf{i}} \in S_{q}^{o}\left[\mathbf{V}_{\text {or, }}, \mathbf{Y} \cup \mathbf{\Upsilon}\right]$. Moreover, the fact that there exists a simple $\mathbf{V}_{\mathbf{i}}-\mathbf{Y}$ path covering an element of $\mathbf{V}_{\mathbf{s}}(\mathbf{q})$ means that there exists a direct $S_{q}^{o}\left[\mathbf{V}_{\text {or, }}, \mathbf{Y} \cup \mathbf{\Upsilon}\right]-\mathbf{Y} \cup \mathbf{\Upsilon}$ path included in $\mathcal{E}_{0} \cup \mathcal{E}_{q}$ and containing at least one edge of $\mathcal{E}_{q}$. Therefore, completed system $\left(\Sigma_{\Lambda}^{c}\right)$ satisfies condition of Proposition 1 and the Proposition 1 follows.

Example 2. Consider now the SSLS represented by the digraph depicted in Figure 2. We have that, $\mathbf{V}_{\text {or }, \mathbf{1}}=\mathbf{V}_{\text {or, }, 2}=$

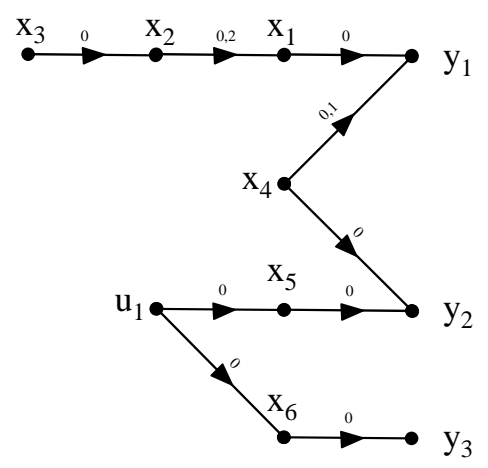

Figure 2. Digraph associated to system of Example 2

$\left\{\mathbf{u}_{\mathbf{1}}, \mathbf{x}_{\mathbf{3}}, \mathbf{x}_{\mathbf{4}}\right\}$ and so $S_{1}^{o}\left[\mathbf{V}_{\mathbf{o r}, \mathbf{1}}, \mathbf{Y}\right]=S_{2}^{o}\left[\mathbf{V}_{\mathbf{o r}, \mathbf{1}}, \mathbf{Y}\right]=$ $\left\{\mathbf{y}_{1}, \mathbf{y}_{2}, \mathbf{y}_{3}\right\}$. Thus, all the direct $S_{1}^{o}\left[\mathbf{V}_{\text {or, }, 1}, \mathbf{Y}\right]-\mathbf{Y}$ paths have a zero length and so condition of Proposition 1 is not satisfied. For mode 1 , only $x_{4}$ is a vertex which begins specific edge and so $\mathbf{V}_{\mathbf{s}}(\mathbf{1})=\left\{\mathbf{x}_{\mathbf{1}}\right\}$. For mode $2, \mathbf{V}_{\mathbf{s}}(\mathbf{1})=\left\{\mathbf{x}_{\mathbf{2}}\right\}$. Therefore, an additional sensor in $\Upsilon=\left\{\mathbf{x}_{4}, \mathbf{x}_{2}, \mathbf{x}_{3}\right\}$ allows to ensure the location observability property.

\subsection{Computational issues}

The conditions of Proposition 1 need few information about the system. Moreover, it is quite easy to check it by means of wellknown combinatorial techniques. Indeed, from a computational point of view, the first step of our procedure requires the calculation of the strongly connected components which can be done using an algorithm which complexity order equals $O(N \log (N))=O(n \log (n))[10]$, where $N=n+m+p$ the number of vertices of our graphs. Note that, without loss of generality, $M=O\left(n^{2}\right)$. After finding the strongly components, we must order these components simply by comparison to find the minimal elements with a $O\left(n^{2}\right)$ complexity order algorithm. The third step is the computation of the output separator between the maximal strongly connected components and the output sets. It is based on the Ford-Fulkerson algorithm which must be executed $n+p$ times and so the complexity order for this step is $n \times O\left(n^{3}\right)=O\left(n^{4}\right)$. To check the location observability condition or to compute the possible additional sensors' locations, we must find all the vertices belonging to direct $S_{q}^{o}\left[\mathbf{V}_{\mathbf{o r}, \mathbf{q}}, \mathbf{Y}\right]-\mathbf{Y}$ paths and extract those which are the begin vertices of specific edges. This is done by successor and predecessor computation algorithms, which have $O\left(n^{2}\right)$ complexity order. Therefore, without any simplification, the complexity order of algorithms using this method is, in the worst case, $O\left(n^{4}\right)$. The fact that the overall complexity order is not exponential makes the proposed method suited to deal with large scale systems. Obviously, some researches can be done in order to lower the overall order complexity by reducing the graph for example or subdividing it, but it is not the aim of the paper.

\subsection{Generalization to multi-mode case}

Since state and input observability of each mode has to be studied separately, the main difficulty is to generalize location observability criterion and more precisely the matrix decomposition related to each mode into two parts: a common one and a specific one.

Consider SSLS $\left(\Sigma_{\Lambda}\right)$ defined in Equation (2), where $Q \stackrel{\text { def }}{=}$ $\{1,2, \ldots, N\}$. We take the following notations: for $q \in$ $\{1,2, \ldots, N\}, q^{\prime} \in\{1,2, \ldots, N\}$ with $q \neq q^{\prime}, A^{\lambda}(q)=$ $A_{q, q^{\prime}}^{\lambda}+A_{q, \overline{q^{\prime}}}^{\lambda}$, where $A_{q, q^{\prime}}^{\lambda}=A_{q^{\prime}, q}^{\lambda}$ denotes the common part between $A^{\lambda}(q)$ and $A^{\lambda}\left(q^{\prime}\right)$ and $A_{q, \overline{q^{\prime}}}^{\lambda}$ represent the specific part of $A^{\lambda}(q)$ relatively to $A^{\lambda}\left(q^{\prime}\right)$. Similarly, $B^{\lambda}(q)=B_{q, q^{\prime}}^{\lambda}+B_{q, \overline{q^{\prime}}}^{\lambda}$, $C^{\lambda}(q)=C_{q, q^{\prime}}^{\lambda}+C_{q, q^{\prime}}^{\lambda}$ and $D^{\lambda}(q)=D_{q, q^{\prime}}^{\lambda}+D_{q, q^{\prime}}^{\lambda}$. We have multiple decompositions for matrices $A^{\lambda}(q), B^{\lambda}(q), C^{\lambda}(q)$ and $D^{\lambda}(q)$ useful only in location observability study. To each of these matrices, we associate, as in Section 3, an edge subset. Thus, we state:

Proposition 2. SSLS $\left(\Sigma_{\Lambda}\right)$ with $N$ modes associated to digraph $\mathcal{G}\left(\Sigma_{\Lambda}\right)$ is generically location observable if for each pair $q \in\{1,2, \ldots, N\}, q^{\prime} \in\{1,2, \ldots, N\}$ with $q \neq q^{\prime}$, Proposition 1 is satisfied by substituting edge subsets $\mathcal{E}_{0}$ by $A_{q, q^{\prime}}$-edges $\cup B_{q, q^{\prime}}$-edges $\cup C_{q, q^{\prime}}$-edges $\cup D_{q, q^{\prime}}$-edges, $\mathcal{E}_{1}$ by $\mathcal{E}_{q, \overline{q^{\prime}}}=A_{q, \overline{q^{\prime}}}$-edges $\cup B_{q, \overline{q^{\prime}}}$-edges $\cup C_{q, \overline{q^{\prime}}}$-edges $\cup D_{q, \overline{q^{\prime}}}$-edges and $\mathcal{E}_{2}$ by $\mathcal{E}_{q^{\prime}, \bar{q}}=A_{q^{\prime}, \bar{q}^{-}}$-edges $\cup B_{q^{\prime}, \bar{q}^{-}}$edges $\cup C_{q^{\prime}, \bar{q}^{-}}$-edges $\cup$ $D_{q^{\prime}, \bar{q}^{-}}$edges.

Proof: The proof is quite immediate starting from Proposition 1 and considering that the location observability in multimode case is equivalent to the distinguishability of each pair of modes. 
In the multi-mode case, concerning the additional sensors placement, we generalize the results of Proposition 1:

Proposition 3. Consider a multi-mode SSLS $\left(\Sigma_{\Lambda}\right)$ with $N$ modes associated to digraph $\mathcal{G}\left(\Sigma_{\Lambda}\right)$. For each, $q \in\{1,2, \ldots, N\}, q^{\prime} \in\{1,2, \ldots, N\}$ with $q \neq q^{\prime}$, let denote by $\mathbf{V}_{\mathbf{s}}\left(\mathbf{q}, \overline{\mathbf{q}^{\prime}}\right)=$ $\left\{\mathbf{v} \in \mathcal{V}, \mathbf{v}\right.$ is the begin vertex of an edge belonging to $\left.\mathcal{E}_{q, \overline{q^{\prime}}}\right\}$ and $\mathbf{V}_{\mathbf{s}}\left(\mathbf{q}^{\prime}, \overline{\mathbf{q}}\right)=\{\mathbf{v} \in \mathcal{V}, \mathbf{v}$ is the begin vertex of an edge belonging to $\left.\mathcal{E}_{q^{\prime}, \bar{q}}\right\}$.

To recover the sufficient condition of Proposition 2, the additional sensors $\Upsilon$ must measure at least one component in each subset $\operatorname{Sens}_{q, q^{\prime}}, q \in\{1,2, \ldots, N\}, q^{\prime} \in\{1,2, \ldots, N\}$ with $q \neq q^{\prime}$ defined by:

Sens $_{q, q^{\prime}}=\left(V_{\text {ess }, q}\left[\mathbf{V}_{\mathbf{o r}, \mathbf{q}}, \mathbf{Y}\right] \cap \quad\left\{\mathbf{v}_{\mathbf{i}}\right.\right.$ s.t. $\exists$ a simple $\mathbf{v}_{\mathbf{i}}-\mathbf{Y}$ path, covering an element of $\left.\left.\mathbf{V}_{\mathbf{s}}\left(\mathbf{q}, \overline{\mathbf{q}^{\prime}}\right)\right\}\right) \cup$

$\left(V_{e s s, q^{\prime}}\left[\mathbf{V}_{\text {or }, \mathbf{q}^{\prime}}, \mathbf{Y}\right] \quad \cap \quad\left\{\mathbf{v}_{\mathbf{i}}\right.\right.$ s.t. $\exists$ a simple $\mathbf{v}_{\mathbf{i}}-\mathbf{Y}$ path covering an element of $\left.\left.\mathbf{V}_{\mathbf{s}}\left(\mathbf{q}^{\prime}, \overline{\mathbf{q}}\right)\right\}\right)$

Proof: From Proposition 1, taking a measure on each set Sens $s_{q, q^{\prime}}$ guarantees the distinguishability of modes $q$ and $q^{\prime}$. Thus, the location observability, which is equivalent to the distinguishability of all the pairs $q \in\{1,2, \ldots, N\}, q^{\prime} \in$ $\{1,2, \ldots, N\}$ with $q \neq q^{\prime}$ is ensured by measuring one component in each subset Sens $_{q, q^{\prime}}, q \in\{1,2, \ldots, N\}, q^{\prime} \in$ $\{1,2, \ldots, N\}$ with $q \neq q^{\prime}$.

\section{CONCLUSION}

In this paper, we propose a graph-theoretic tool to analyze generic mode location observability for switching structured linear systems with unknown inputs. At first, we provide a simple but not trivial sufficient condition to ensure the generic location observability of switching structured linear systems. Next, when this observability condition is not satisfied, we study the location of additional sensors in order to recover the generic location observability. This problem has not been solved previously in the literature. It is shown also that our approach uses classical programming techniques and is free from numerical difficulties since it uses well-known combinatorial techniques. Indeed, all the algorithms used in the paper have polynomial complexity orders. This makes our proposed method well-suited to large scale systems.

In future works, we will interest to complete this study by providing quite simple necessary and sufficient location observability conditions and also a more complete procedure recovering this property when it is not achieved. We will interest also in coupling the presented approach based on a structural criterion (here the location observability) with reliability indicators and economic cost functions to propose a global strategy to design large scale systems.

\section{REFERENCES}

[1] P. Antsaklis. Hybrid systems: Theory and applications. Proceedings of the IEEE, 88(7 Special Issue):879 - 887, 2000.

[2] M. Babaali and M. Egerstedt. On the observability of piecewise linear systems. In IEEE Conference on Decision and Control, pages 26-31, Bahamas, 2004.

[3] M. Babaali and G. J. Pappas. Observability of switched linear systems in continuous time. In M. Morari and L. Thiele, editors, Hybrid Systems: Computation and Control, volume 3414 of Lecture Notes in Computer Science, pages 103-117. Springer, Berlin, 2005.
[4] A. Bemporad, G. Ferrari-Trecate, and M. Morari. Observability and controllability of piecewise affine and hybrid systems. IEEE Transactions on Automatic Control, 45(10):1864-1876, 2000.

[5] S. Chaib, D. Boutat, A. Benali, and J-P. Barbot. Observability of the discrete state for dynamical piecewise hybrid systems. Nonlinear Analysis, 63(3):423-438, 2005.

[6] P. Collins and J. H. Van Schuppen. Observability of piecewise-affine hybrid systems. In R. Alur and G. J. Pappas, editors, Hybrid Systems: Computation and Control, volume 2993 of Lecture Notes in Computer Science, pages 265-279. Springer, Berlin, 2004.

[7] E. De Santis, M. D. Di Benedetto, and G. Pola. Observability of internal variables in interconnected switching systems. In IEEE Conference on Decision and Control, pages 4121-4126, San Diego, USA, 2006.

[8] E. De Santis, M. D. Di Benedetto, and G. Pola. Observability and detectability of linear switching systems: A structural approach. arXiv:0802.4045v1 [math.DS], submitted on 2008.

[9] J. M. Dion, C. Commault, and J. W. van der Woude. Generic properties and control of linear structured systems: a survey. Automatica, 39(7):1125-1144, 2003.

[10] L. K. Fleischer, Bruce Hendrickson, and A. Pinar. On Identifying Strongly Connected Components in Parallel, pages 505-511. Lecture Notes in Computer Science. Springer Berlin / Heidelberg, 2000.

[11] R. Johansson and A. Rantzer (Eds). Hybrid systems in automotive control. International Journal of Control, 80(11, Special issue), 2007.

[12] K. Murota. System Analysis by Graphs and Matroids. Springer-Verlag, New York, U.S.A., 1987.

[13] L. Pina and A. Botto. Simultaneous state and input estimation of hybrid systems with unknown inputs. Automatica, 42:755-762, 2006.

[14] K. J. Reinschke. Multivariable Control. A Graph Theoretic Approach. Springer-Verlag, New York, U.S.A., 1988.

[15] L. M. Silverman. Inversion of multivariable linear systems. IEEE Transactions on Automatic Control, AC 14(3):270-276, 1969.

[16] A.J. van der Schaft and H. Schumacher. An introduction to hybrid dynamical systems. In M. Thoma, editor, Lecture Notes in Control and Information Sciences, volume 251. Springer, Berlin / Heidelberg, 2000.

[17] J. W. van der Woude. The generic number of invariant zeros of a structured linear system. SIAM Journal of Control and Optimization, 38(1):1-21, 2000.

[18] J. W. van der Woude, C. Commault, and J. M. Dion. Zero orders and dimensions of some invariant subspaces in linear structured systems. Mathematics of Control, Signals and Systems, 16(2-3):225-237, 2003.

[19] R. Vidal, A. Chiuso, and S. Soatto. Observability and identifiability of jump linear systems. In IEEE Conference on Decision and Control, pages 3614-3619, Las Vegas, USA, 2002.

[20] R. Vidal, A. Chiuso, S. Soatto, and S.S. Sastry. Observability of linear hybrid systems. In O. Maler and A. Pnueli, editors, Hybrid Systems: Computation and Control, volume 2623 of Lecture Notes in Computer Science, pages 526-539. Springer, Berlin, 2003.

[21] L. Vu and D. Liberzon. On invertibility of switched linear systems. In IEEE Conference on Decision and Control, pages 4081-4086, San Diego, USA, 2006. 